\title{
LHX6 is essential for the migration of human pluripotent stem cell-derived GABAergic interneurons
}

\section{Dear Editor,}

GABAergic interneurons (Gls) play an essential inhibitory role in regulating neural circuitry and neurological activities. Approximately $54 \%$ GABAergic interneuron population originated from medial ganglionic eminence (MGE) at E13.5 in rodents (Butt et al., 2005), and migrated from MGE to dorsal cortex tangentially starting at E13-14 after the GABAergic neurons become postmitotic. Dysfunction of GABAergic interneurons causes severe neurological diseases, such as autism, schizophrenia and depression. Recent years, many studies have shown transplantation of Gls improved the neuronal function of animal models such as epilepsy, neuropathic pain, and fear erasure (Bráz et al., 2012; Cunningham et al.; Yang et al., 2016). Since migration is the intrinsic nature of Gls, regulation of Gls migration could be essential for cell therapy. However, the mechanism of human Gls migration remains unknown, which brings difficulties to control cell migration after transplantation.

According to the rodent studies, numerous transcriptional factors are involved in regulating Gls migration, such as DIx1/2, Nkx2.1, and Lhx6. LIM Homeobox 6 (Lhx6) is a subtype gene of LIM homeodomain family. During early development, Lhx6 is expressed in the MGE and plays an essential role in the migration of Gls from MGE to the cortex in mice (Liodis et al., 2007; Zhao et al., 2008). Migration deficiency of Gls is found in Ihx6-null mutant mice (Flandin et al., 2011). However, the role of the LHX6 in human Gls migration have not been investigated yet. Regulation of migration related transcriptional factors provide us a new prospective to promote Gls migration in cell therapy.

In order to study the role of LHX6 in modulating the migration of human GABAergic interneurons (Gls), we constructed LHX6 knockout hESC lines and differentiated into Gls. We constructed LHX6 knockout hPSC lines by using a donor-free paired gRNA-guided CRISPR/Cas9 strategy (Chen et al., 2015) (Fig. 1A). LHX6 knockout (KO) hESC colonies were used according to the results of DNA-sequencing (Fig. 1B). During GABA interneuron differentiation, the mRNA level of $L H X 6$ was dramatically decreased at day 17 , and the expression of SHH was decreased in LHX6 KO cells whereas the mRNA levels of $N K X 2.1$ and $L H X 8$ did not change after knock out of $L H X 6$ (Fig. S1B), which is reasonable that cells in the MGE mantle area could enhance SHH signaling (Flandin et al., 2011). By day 22, the LHX6 KO hESCderived GI progenitors showed similar expression of NKX2.1 compared to controls by using immunostaining, while an increased level of COUPTFII, a transcription factor of the caudal ganglionic eminence (CGE) (Fig. S1A and S1C). In addition, OLIG2, a subventricular zone marker of the MGE area, was significantly decreased in the LHX6 knockout group, while the FOXG1 was not altered (Fig. S1A and S1C).

By day 35 , the percentage of GABA+ cells and GAD67+ cells in total cells was around $40 \%$ in $\mathrm{KO}$, while around $80 \%$ in control group, indicating knockout of LHX6 decreased the Gls differentiation from hESCs (Fig. 1C and 1D). Strikingly, the explant experiment demonstrated that the LHX6 knockout GABA interneurons exhibited reduced migratory ability after plating for $24 \mathrm{~h}$ (Fig. 1E and 1F), indicating that LHX6 was crucial for the migration of GABA interneurons.

Since LHX6 KO suppressed Gls migration, we determined to further explore if LHX6 overexpression (OE) could promote Gls migration. LHX6 conditional overexpression human pluripotent stem cell (hPSCs) lines was constructed as our previous report (Yuan et al., 2018). Gls were robustly differentiated in both LHX6 OE and control group. SAG, an agonist of sonic hedgehog signaling pathway was used for ventral patterning (Fig. 1G). By 3 weeks of differentiation, over $80 \%$ of hPSC-derived cells expressed NKX2.1 (Fig. S2A and S2B), indicating the cell identity of medial ganglionic eminence (MGE) progenitors. By day $35,90 \%$ of cells were TUJ1+ and $75 \%$ of cells were GABA+ (Fig. S2A and S2B) in both groups.

In retrospect of the RNA-seq profiles of LHX6 overexpression Gls (Yuan et al., 2018), we found migration-related GO terms were highly enriched in LHX6 OE Gls compared with control group (Fig. S2C). According to GO annotation of biological process, positive regulation of cell motility-related terms were enriched in LHX6 OE group. In addition, numerous genes associated with positive cell migration such as SYNE2, SEMA4D, TIAM1 (Tanaka et al., 2004; Zhang et al., 2009; Kuzirian et al., 2013) were increased following 
A

CRISPR/CAS9

LHX6 KO hPSCS

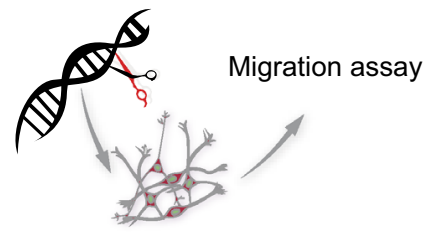

LHX6 KO GINs
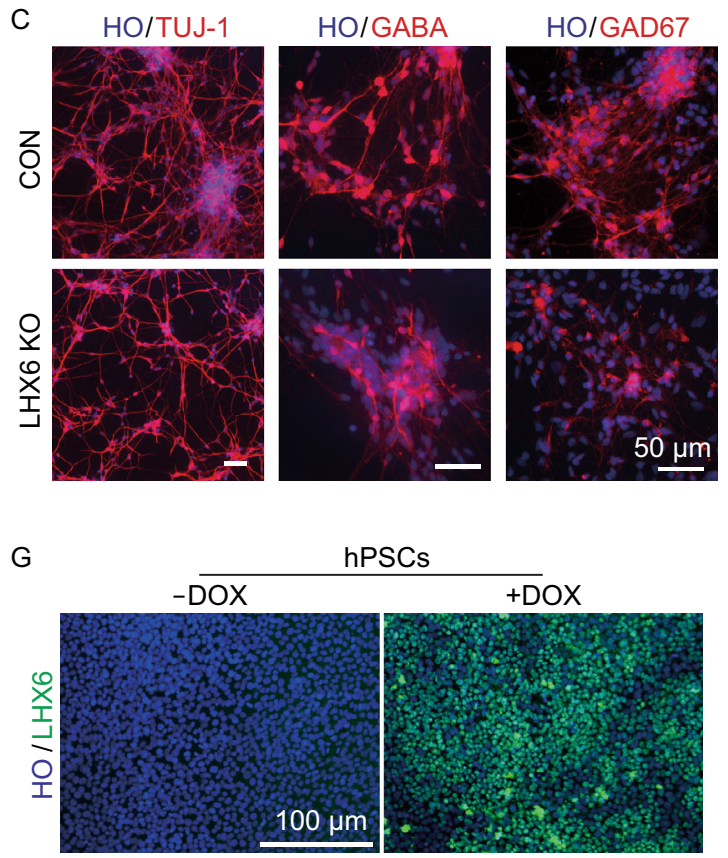

hPSCs


Primers-L

LHX6-EXON2

Primers- $\mathrm{R}$

g'R̄NĀ-ĹL

gRNÄ-R - - . - . - .

WT: GGGAGATTTCGTTGTCAATTGGTGCTCGGGGGCC----266bp----GGGGTAGGTGTTGCGTGCGTGTTGTCGGGGCGTGGGACGCA KO4a: GGGAGATTTCGTTGTCAATTGGT—TGTCGGGGCGTGGGACGCA $298 \mathrm{bp}$ KO4b: GGGAGATTTCGTTGTCAATTGG -GTCGGGGCGTGGGACGCA -300 bp
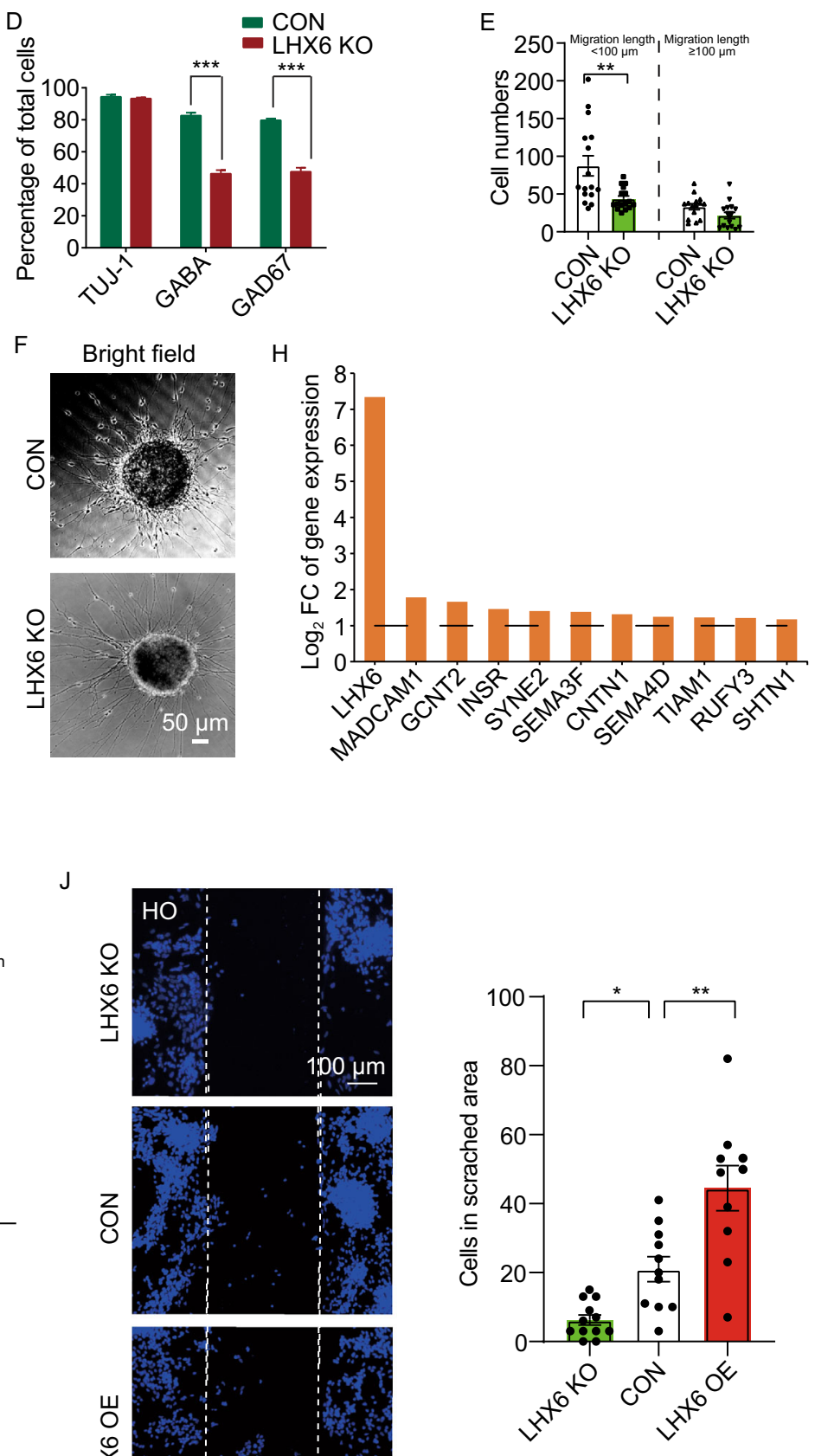
Figure 1. LHX6 regulated human Gls migration in vitro. (A) Schematic diagram of the analysis of Gls derived from LHX6 KO hPSCs. (B) Genomic DNA analysis for two LHX6 KO colonies. (C) Representative images of TUJ-1, GABA and GAD67 expressing in CON and LHX6 KO group. (D) Quantification of TUJ-1, GABA and GAD67 in CON and LHX6 KO group. ( $E$ and F) The LHX6 KO hESC-derived Gls showed decreased migration ability in an explant migration assay after attachment for $24 \mathrm{~h}$. Scale bar, $50 \mu \mathrm{m}$. Quantification of migration in $(F)$. At least 15 neurospheres were counted from each group, $n \geq 3$ for each group. Bar graphs were presented as mean \pm SEM. (G) Immunostaining of LHX6 in hPSCs after doxycycline treatment; $(\mathrm{H})$ Fold change of migration related factors. (I) LHX6 OE hESC-derived Gls showed increased migration ability in an explant migration assay after attachment for $24 \mathrm{~h}$. Quantification of migration at right panel. (J) Hoechst staining of hPSC-derived Gls in the control, LHX6 KO and LHX6 OE group in the scratch assay. The right panel is the quantification of the percentage of cells that migrated into the scratch area after 1 day post-attachment. Bar graphs were presented as mean \pm SEM; $n \geq 3$.

LHX6 overexpression (Fig. $1 \mathrm{H}$ ). These results demonstrated that overexpression of LHX6 may positively promote GIs migration in gene profile.

To ascertain whether overexpression of LHX6 regulates migration of human Gls, we performed explant assay after attachment. On one day post-attachment, the LHX6 OE GIs showed a significant increasement in the number of cells that migrated more than $100 \mu \mathrm{m}$ distance, in comparison with the control group (Fig. 1I). To further confirm the effect of LHX6 on cell migration in vitro, we cultured the Gls as single cells and performed scratch assay to determine the migratory ability. A few more cells migrated to scratched area in LHX6 compared with control group and fewer cells migrated in LHX6 KO group (Fig. 1J).

To further confirm that LHX6 regulates the migration in human Gls, we injected 5,000 7-week hPSC-derived GI progenitors onto the MGE area of embryonic day 15 (E15) mouse brain slices (Fig. 2A). After two weeks of co-culture, extensive ventral to dorsal migration was observed in LHX6 overexpression group in the direction of the MGE to the cortex. Human nuclei $(\mathrm{hN})$ was used to label the grafted cells. We detected $\mathrm{hN}$ and GABA co-labeled cells in both groups. Notably, in similar areas of the brain slices, a dramatically increased number of $\mathrm{hN}$ cells were found in LHX6 overexpression group (Fig. 2B-D). Moreover, human GABAergic interneurons also showed an increased number in LHX6 OE group (Fig. S4). A distinct migration trace clearly showed that the grafted cells migrated from the injection site to the cerebral cortex in LHX6 overexpression transplants (Fig. 2E). To analyze the migration, the brain slices were divided into five areas based on the distance from the transplantation sites (Fig. 2F). At a distance of $0.75-2.25$ $\mathrm{mm}$, the number of migrated cells were similar in the two groups (+/- DOX). Significantly, more LHX6 overexpression GABA interneurons migrated into the area that was 2.25-3 $\mathrm{mm}$ away from the injection site (Fig. $2 \mathrm{~F}$ and $2 \mathrm{G}$ ).

To more systematically test the role of LHX6 in migration, we used fused forebrain organoids to determine the $\mathrm{GI}$ migration from ventral to dorsal (Fig. $2 \mathrm{H}$ ). Cerebral organoids and ventral MGE organoids were generated from hPSCs by following the reported protocols with slight modification, which mimic the endogenous developmental environment more closely than 2D culture (Birey et al., 2017) (Fig. S3A). To induce region-specific brain organoid, cerebral organoids were generated by default and ventral forebrain organoids were patterned with SAG, respectively. After 60 days culture from hPSCs, the cerebral organoids exhibited multiple cortical layer-like structures, with expressing cortical markers PAX6, TBR1, CTIP2, as well as neural progenitor markers SOX2 and NESTIN (Fig. S3B). 5 weeks old ventral forebrain organoids expressed NKX2.1, GABA and GAD67 (Fig. S3C). One week before fused, ventral organoids (OE, control and $\mathrm{KO}$, respectively) were labeled with GFP by lentivirus infection, and we then fused the cerebral and ventral organoids in a well of 96-well plate for 3 days (Fig. 2I). As a result of this experiment, more GFP+ cells migrated from ventral organoids to dorsal organoids in LHX6 OE group (Fig. S3D).

In pioneering studies, transplantation of hPSCs derived Gls was reported to reduce seizure activities and held promise for neuropsychiatric diseases (Cunningham et al. 2014). To further investigate the migration of grafted human GABA interneurons in vivo, we transplanted the 7-week hPSCs-derived MGE progenitors into the ventral forebrain of severe combined immunodeficiency (SCID) neonatal mice (Fig. 2J). Both LHX6 OE group and control group were transplanted. After 3 months post-transplantation, most grafted cells differentiated into Gls that expressing GABA in human nuclei ( $\mathrm{hN}$ ) positive cells (Fig. 2K). Besides, a few grafted cells migrated from injected site (basal forebrain) to the dorsal cortex after 3 months (Fig. 2L). After transplantation, cells in LHX6 OE group were more likely migrated to superficial layers (Fig. 2M). Notably, the LHX6 overexpressed Gls showed increased migration ability and exhibited increased number of migrated cells in each layer of the cortex, whereas the control Gls showed fewer cells in the layer I-IV (Fig. 2L and 2M), indicating LHX6 overexpression significantly enhanced the cell migration to dorsal cortical layers in vivo.

Along with the technological advancement, Gls could be generated with high efficiency in vitro. Consequently, Gls transplantation has shown great therapeutic potential for many neurological disorders. Grafted cells might functionally integrate into host circuits and modify the activity of host cells after migration. However, very little was found in the regulation of human GABA interneurons migration after transplantation. Based on hPSC neural differentiation, we describe the role of LHX6 in human GABA interneuron migration by using knockout/overexpression strategies. In 
A

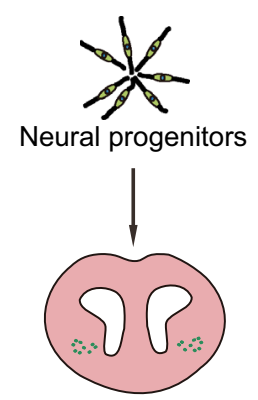

Brain slice co-culture
B

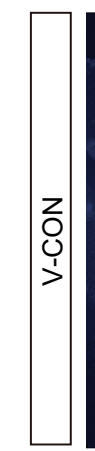

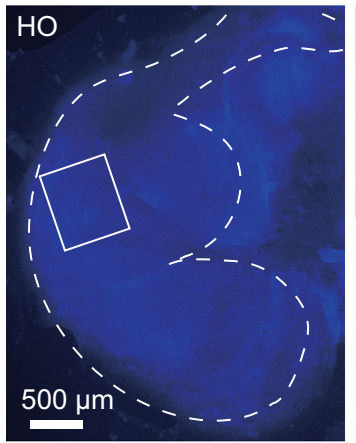

$500 \mu \mathrm{m}$


D

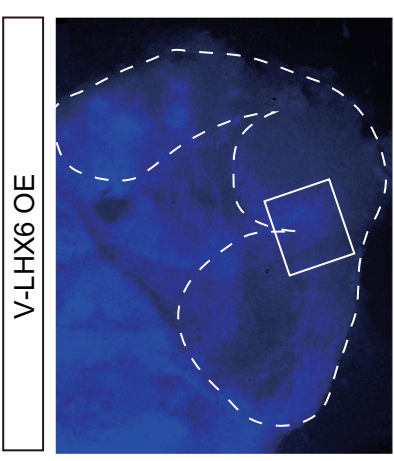

E

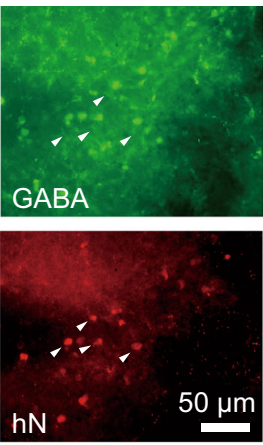

C

Migration trace (V-LHX6 OE)

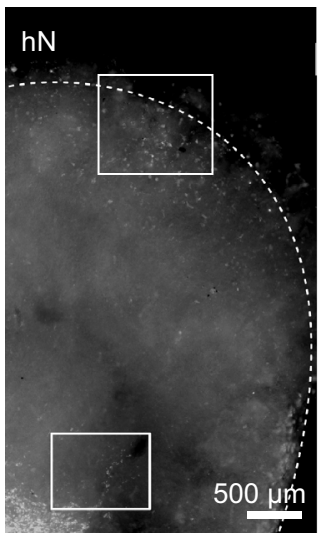

F

Dorsal forebrain

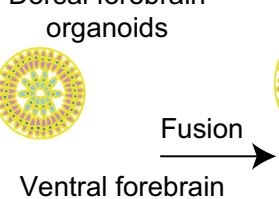
organoids

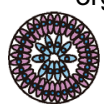

Migration analysis


G
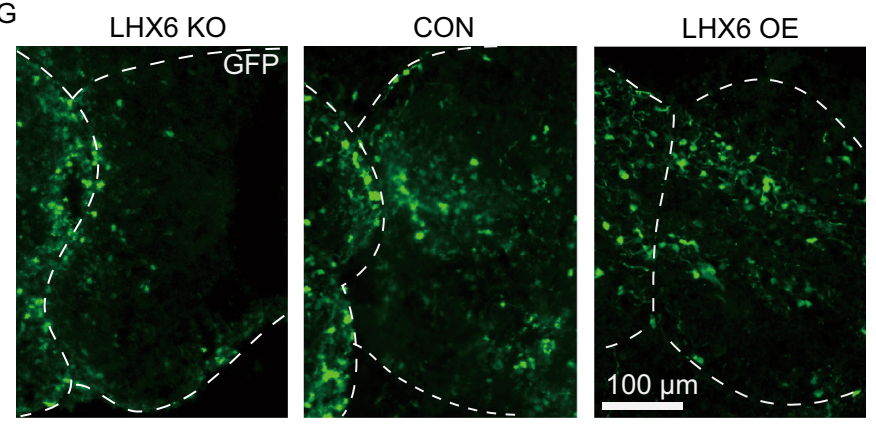

K

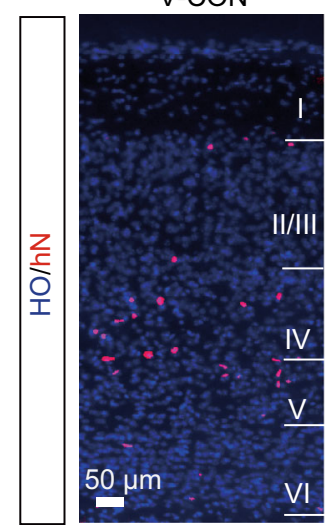

V-LHX6 OE

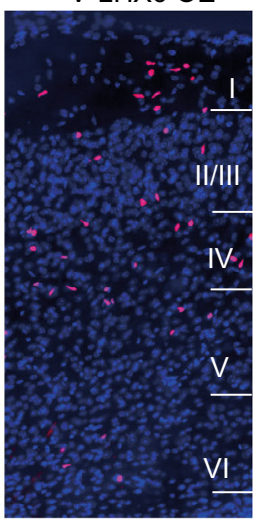

L

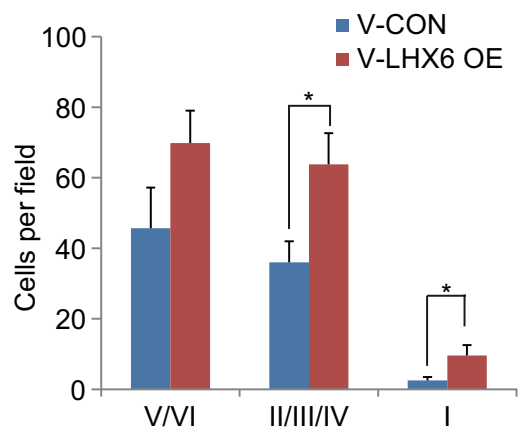


Figure 2. Human Gls migration was promoted by LHX6 OE in brain slice co-culture and in vivo transplantation. (A) Schematic representing mouse brain slices and the hPSC-derived Gls co-culture experiment. Human Gls were injected into the MGE area of E15 brain slices. (B-D) Mouse brain slices were co-cultured with control and LHX6 OE hPSCderived Gls. The insets show similar areas of the brain slice, and the co-cultured slices were labeled with $\mathrm{hN}$ (human nuclei). (E) The migration trace of LHX6 OE cells. The white dashed arrow shows the migration trace of $\mathrm{hN}+$ cells moving from the MGE to the cortex. (F) Schematic showing the division of the brain slices into different distances from the transplantation site. (G) Quantification of the numbers of $\mathrm{hN}+$ cells at different distances in the co-cultured brain slices; $n=6$ for $\mathrm{V}$-CON and $n=5$ for V-LHX6 OE. (H) Schematic diagram of fused forebrain organoids. Hoechst staining of fused organoids. Left side was a 35-day ventral forebrain organoid, right side was a 35-day dorsal forebrain organoid. (I) Cells labeled with GFP migrated from ventral organoids (left) to dorsal organoids (right). Quantification in (Fig. S3D). For each group, $n \geq 5$. (J) Schematic showing the transplantation of hPSC-derived GABAergic progenitors into the basal forebrain of neonatal mice. (K) Immunostaining image of $\mathrm{hN}$ and GABA co-labeling. (L) Migrated $\mathrm{hN}+$ cells in the cortical layers in the control and LHX6 OE groups. Scale bar, $50 \mu \mathrm{m}$. (M) Quantification of $\mathrm{hN}+$ cells in each of the cortical layers in the control and LHX6 OE groups; $n=4$ for $\mathrm{V}-\mathrm{CON}$ and $n=5$ for V-LHX6 OE.

this study, we found that LHX6 is critical for controlling human GABAergic interneuron migration, which is confirmed with previous studies from $I h x 6$ transgenic rodents (Liodis et al., 2007). The GABAergic interneurons exhibited enhanced migration ability in cell culture, brain slice co-culture, cell transplantation and fused forebrain organoids upon overexpression of LHX6, while decreased migration ability with LHX6 knockout.

In our current study, LHX6 knockout cell line showed a decreased Gls differentiation efficiency (decreased GABA and GAD67 percentage), while in rodent studies that the GABA neuron number is similar in Ihx6 KO mice (Liodis et al., 2007). The percentage of COUPTFIl positive cells increased in the LHX6 KO cell line, whereas decreased in the LHX6 overexpression cell lines in our previous observation (Yuan et al., 2018). Correspondingly, Ihx6 ko mice studies also showed Lhx6 could repress CGE-identity in MGE cells (Vogt et al., 2014). Therefore, our studies indicated LHX6 is important for the cell fate of ventral precursors and the generation of human GABA interneurons. We also used fused forebrain ventral-dorsal organoids to analyze human cortical interneuron migration, which is a comparatively ideal model to study human forebrain development. An interesting phenomenon was the size of dorsal organoids was vitally larger than ventral organoids (data not shown), suggesting the human cortical neural precursors may have stronger proliferative ability than ventral neural precursors. The result confirmed that LHX6 enhanced cell migration, and moreover, it applied a dynamic model for analyzing different human brain region interactions. Delayed migration of tangentially migrating Gls was found in Lhx6-deficient embryos (Liodis et al., 2007). Rodent studies showed that Ihx6 could directly promote the expression of CXCR4/7 to form normal Gls migration (Vogt et al., 2014). However, in our RNA-seq data, CXCR4 showed no significant change and CXCR7 even downregulated in LHX6 OE group. It suggests human Gls migration might be regulated by a different and complex manner.

In conclusion, our results demonstrated that LHX6 is an essential transcription factor for the migration of human GABA interneurons by using knockout and overexpression strategies in human pluripotent stem cells. This method let us dig LHX6 function in human neurodevelopment from a new perspective. It could also be an important guide for the application of cell therapy in related diseases.

\section{FOOTNOTES}

We thank Dr. Su-Chun Zhang (Waisman Center and WiCell Research Institute at the University of Wisconsin) for kindly providing the AAVS1-TALEN plasmids and crispr-cas9 plasmids. This study was supported by the Strategic Priority Research Program of the Chinese Academy of Sciences (Grant No. XDA16010306), the National Natural Science Foundation of China Grants (Grant Nos. 81922022, 91849117, and 81471301), the National Key Research and Development Program of China (2019YFA0802703 and 2016YFC1306703), the Jiangsu Outstanding Young Investigator Program (BK20160044), and the Jiangsu Province's Innovation Program, Postgraduate Research and Practice Innovation Program of Jiangsu Province.

Fang Yuan, Kai-Heng Fang, Yuan Hong, Shi-Bo Xu, Min Xu, Yufeng Pan and Yan Liu declare that they have no conflict of interest. All institutional and national guidelines for the care and use of laboratory animals were followed. This article does not contain any studies with human or animal subjects performed by the any of the authors.

Fang Yuan ${ }^{1}$, Kai-Heng Fang ${ }^{1}$, Yuan Hong ${ }^{1}$, Shi-Bo $\mathrm{Xu}^{1}$, Min $\mathrm{Xu}^{1}$, Yufeng Pan ${ }^{2}$, Yan Liu ${ }^{1,3,4 \bowtie}$

\footnotetext{
${ }^{1}$ Institute for Stem Cell and Neural Regeneration, School of Pharmacy, Nanjing Medical University, Nanjing 211166, China

${ }^{2}$ Key Laboratory of Developmental Genes and Human Disease, Institution of Life Sciences, Southeast University, Nanjing 2100096, China

${ }^{3}$ State Key Laboratory of Reproductive Medicine, Nanjing Medical University, Nanjing 211166, China

${ }^{4}$ Co-innovation Center of Neuroregeneration, Nantong University, Nantong 226019, China

$\bowtie$ Correspondence: yanliu@njmu.edu.cn (Y. Liu)
} 


\section{OPEN ACCESS}

This article is licensed under a Creative Commons Attribution 4.0 International License, which permits use, sharing, adaptation, distribution and reproduction in any medium or format, as long as you give appropriate credit to the original author(s) and the source, provide a link to the Creative Commons licence, and indicate if changes were made. The images or other third party material in this article are included in the article's Creative Commons licence, unless indicated otherwise in a credit line to the material. If material is not included in the article's Creative Commons licence and your intended use is not permitted by statutory regulation or exceeds the permitted use, you will need to obtain permission directly from the copyright holder. To view a copy of this licence, visit http:// creativecommons.org/licenses/by/4.0/.

\section{REFERENCES}

Birey F, Andersen J, Makinson CD, Islam S, Wei W, Huber N, Fan HC, Metzler KR, Panagiotakos G, Thom N et al (2017) Assembly of functionally integrated human forebrain spheroids. Nature 545:54

Bráz JM, Sharif-Naeini R, Vogt D, Kriegstein A, Alvarez-Buylla A, Rubenstein JL, Basbaum Al (2012) Forebrain GABAergic neuron precursors integrate into adult spinal cord and reduce injuryinduced neuropathic pain. Neuron 74(4):663-675

Butt SJ, Fuccillo M, Nery S, Noctor S, Kriegstein A, Corbin JG, Fishell $G$ (2005) The temporal and spatial origins of cortical interneurons predict their physiological subtype. Neuron 48 (4):591-604

Chen Y, Cao J, Xiong M, Petersen AJ, Dong Y, Tao Y, Zhang SC (2015) Engineering human stem cell lines with inducible gene knockout using CRISPR/Cas9. Cell Stem Cell 17(2):233-244

Cunningham M, Cho JH, Leung A, Savvidis G, Ahn S, Moon M, Lee PK, Han JJ, Azimi N, Kim KS et al (2014) hPSC-derived maturing
GABAergic interneurons ameliorate seizures and abnormal behavior in epileptic mice. Cell Stem Cell 15(5):559-573

Flandin P, Zhao Y, Vogt D, Jeong J, Long J, Potter G, Westphal H, Rubenstein JL (2011) Lhx6 and Lhx8 coordinately induce neuronal expression of Shh that controls the generation of interneuron progenitors. Neuron 70(5):939-950

Kuzirian MS, Moore AR, Staudenmaier EK, Friedel RH, Paradis S (2013) The class 4 semaphorin Sema4D promotes the rapid assembly of GABAergic synapses in rodent hippocampus. J Neurosci 33(21):8961-8973

Liodis P, Denaxa M, Grigoriou M, Akufo-Addo C, Yanagawa Y, Pachnis V (2007) Lhx6 activity is required for the normal migration and specification of cortical interneuron subtypes. J Neurosci 27(12):3078-3089

Tanaka M, Ohashi R, Nakamura R, Shinmura K, Kamo T, Sakai R, Sugimura $H$ (2004) Tiam1 mediates neurite outgrowth induced by ephrin-B1 and EphA2. Embo J 23(5):1075-1088

Vogt D, Hunt RF, Mandal S, Sandberg M, Silberberg SN, Nagasawa T, Yang Z, Baraban SC, Rubenstein JL (2014) Lhx6 directly regulates Arx and CXCR7 to determine cortical interneuron fate and laminar position. Neuron 82(2):350-364

Yang WZ, Liu TT, Cao JW, Chen XF, Liu X, Wang M, Su X, Zhang $S Q$, Qiu BL, Hu WX et al (2016) Fear erasure facilitated by immature inhibitory neuron transplantation. Neuron 92(6):13521367

Yuan F, Chen X, Fang KH, Wang Y, Lin M, Xu SB, Huo HQ, Xu M, Ma L, Chen $Y$ et al (2018) Induction of human somatostatin and parvalbumin neurons by expressing a single transcription factor LIM homeobox 6. eLife 7:e37382

Zhang X, Lei K, Yuan X, Wu X, Zhuang Y, Xu T, Xu R, Han M (2009) SUN1/2 and Syne/Nesprin-1/2 complexes connect centrosome to the nucleus during neurogenesis and neuronal migration in mice. Neuron 64(2):173-187

Zhao Y, Flandin P, Long JE, Cuesta MD, Westphal H, Rubenstein JLR (2008) Distinct molecular pathways for development of telencephalic interneuron subtypes revealed through analysis of Lhx6 mutants. J Comp Neurol 510(1):79-99
Electronic supplementary material The online version of this article (https://doi.org/10.1007/s13238-019-00686-6) contains supplementary material, which is available to authorized users.

Fang Yuan and Kai-Heng Fang have equally contributed to this work. 